\title{
ISÓTOPOS ESTÁVEIS E PRODUÇÃO DE BEBIDAS: DE ONDE VEM O CARBONO QUE CONSUMIMOS?
}

\author{
Ana Cristina B. Oliveira², Cleber I. SALIMON², Débora Fernandes CALHEIROS ${ }^{2}$, \\ Fernando Antonio FERNANDES ${ }^{2}$, Ivan VIEIRA² ${ }^{2}$ Luiz Fernando CHARBEL ${ }^{2}$, Luiz Fernando Pires ${ }^{2}$, \\ Marcos S.M.B. SALOMÃO ${ }^{2}$, Sandra Furlan NOGUEIRA², Simone VIEIRA², \\ Marcelo Zacharias MOREIRA ${ }^{3}$, Luiz Antônio MARTINELLI ${ }^{3}$, Plínio Barbosa de CAMARGO ${ }^{3, *}$
}

\begin{abstract}
RESUMO
A composição isotópica do carbono $\left(\delta^{13} \mathrm{C}\right)$ foi utilizada na determinação da origem botânica (C3 x C4) de amostras de café, vinho, cerveja e vodka, de diferentes marcas e procedências, comercializadas no Brasil. Dentre as marcas de café analisadas, apenas uma apresentou um elevado percentual de plantas $\mathrm{C} 4 \mathrm{em}$ sua composição, evidenciado pelo valor de $\delta^{13} \mathrm{C}\left(-16,2 \%\right.$ o). Os valores de $\delta^{13} \mathrm{C}$ das amostras de vinhos brasileiros, variaram entre $-25,1$ e $-17,1 \%$, indicando a presença de carbono de origem $\mathrm{C} 4$ nas amostras que tiveram os maiores valores de $\delta^{13} \mathrm{C}$. Duas marcas de vinhos importados, por sua vez, apresentaram $\delta^{13} \mathrm{C}$ característico de planta C3 $(-27,1 \mathrm{e}-26,3 \%$ o). Os valores de $\delta^{13} \mathrm{C}$ das vodkas importadas variaram entre -26,4 e -23,9\%o, e as brasileiras entre -12,8 e - 11,8\%o, excetuando-se uma marca que apresentou valor $-23,1 \%$. As marcas de cervejas importadas, em sua maioria, apresentaram valores mais negativos (-27,3 a $20,7 \%$ o) que as nacionais $(-25,9$ a $-18,4 \%$ o). Estes resultados evidenciam a eficiência desta metodologia na determinação da origem do C e percentual de mistura destas bebidas.

Palavras-chave: isótopo estável; carbono; café; vinho; vodka; cerveja.
\end{abstract}

\section{SUMMARY}

STABLE ISOTOPES AND BEVERAGE PRODUCTION: WHERE DOES THE CARBON WE DRINK COME FROM? We used the carbon isotopic composition $\left(\delta^{13} \mathrm{C}\right)$ to determine the botanical origin (whether $\mathrm{C} 3$ or $\mathrm{C} 4$ ) of coffee, wine, beer and vodka samples from different brands and origins commercialized in Brazil. Only one coffee sample showed a $\delta^{13} \mathrm{C}$ typical of a mixture with $\mathrm{C} 4$ plants $\left(\delta^{13} \mathrm{C}-16.2 \%\right.$ ). The Brazilian wine samples varied from $\delta^{13} \mathrm{C}-25.1$ to $-18.6 \%$, indicating the presence of carbon from $\mathrm{C} 4$ plants in samples with greater values, while the imported wine showed $\delta^{13} \mathrm{C}$ characteristic of $\mathrm{C} 3$ plants $\left(-27.1\right.$ and $-26.3 \%$ ). Imported vodka also showed $\delta^{13} \mathrm{C}$ typical of $\mathrm{C} 3$ plants $(-26.4$ and $-23.9 \%$ o), while Brazilian counterparts were typically made from C4 plants $(-12.8$ to $-11.8 \%$ o), except for one sample (23,1\%o). Most of the imported beers showed more negative $\delta^{13} \mathrm{C}(-27.3$ to $-20.7 \%$ o) than did the Brazilian beers $(-25.9$ to $-18.4 \%$ o). These results demonstrate the method's efficiency for tracing the carbon origin and determining the extent of blending in beverages.

Keywords: stable isotope; carbon; coffee; wine; vodka; beer.

\section{1 - INTRODUÇÃO}

No controle e inspeção de alimentos são utilizadas várias metodologias de análise de qualidade e determinação da composição de acordo com as necessidades regulamentares especificas para cada alimento.

$\mathrm{O}$ uso da razão isotópica do carbono $\left({ }^{13} \mathrm{C} /{ }^{12} \mathrm{C}\right)$, tem sido largamente empregado. A metodologia isotópica é especialmente útil quando a composição de bebidas e alimentos baseia-se em misturas de compostos produzidos a partir de plantas do tipo $\mathrm{C} 3$ e $\mathrm{C} 4$, pois existe uma grande diferença entre a composição isotópica destes dois tipos de plantas. Por exemplo, utiliza-se a composição isotópica para se detectar a adição de açúcar de cana (uma planta $\mathrm{C} 4$ ) durante o processo de fermentação (chaptalização) ou adição de álcool produzido a partir de cana em vinhos e brandies $[8,10,11,13$, $15,18]$. O mesmo princípio é utilizado para detecção de açúcares de origem $\mathrm{C} 4$ em sucos de frutas naturais [5,

1. Recebido para publicação em 06/07/2001. Aceito para publicação em 06/03/2002.

2. Alunos do curso de pós-graduação do Centro de Energia Nuclear na Agricultura - Universidade de São Paulo. Av. Centenário, 303, Caixa Postal 96, Piracicaba, CEP 13400-970 SP.

3. Professores do Laboratório de Ecologia Isotópica do Centro de Energia Nuclear na Agricultura - Universidade de São Paulo. Av. Centenário, 303, Caixa Postal 96, Piracicaba, CEP 13400-970 SP.

* A quem a correspondência deve ser enviada.
$9,11,16]$ e adulteração de amostras de mel com açúcares $\mathrm{C} 4$ comerciais tanto produzidos a partir da cana como de milho, que também é uma planta C4 [4, 14, 19, 20].

A importância que esta metodologia vem assumindo pode ser avaliada pelo fato da mesma ser a metodologia oficial empregada nos Estados Unidos da América nas análises de controle de qualidade de pureza do mel e sucos de frutas naturais. Além disso, a União Européia, com a finalidade de detectar adição de açúcares comerciais no mosto, introduziu a partir de 1990 [6], um rígido controle da qualidade dos vinhos baseado na análise isotópica do etanol e da água extraídos de vinhos produzidos pelos paises da comunidade européia.

No Brasil, os isótopos estáveis são pouco empregados no controle de adulteração de bebidas [14]. Visando mostrar as potencialidades dessa metodologia em nosso país, determinamos a composição isotópica de algumas amostras de café, vinho, cerveja e vodka, de diferentes marcas e procedências, comercializadas no Brasil.

\section{MATERIAL E MÉTODOS}

A composição isotópica do carbono é expressa pela fórmula:

$\delta^{13} \mathrm{C}=\left(\frac{R_{\text {amostra }}-R_{\text {padrào }}}{R_{\text {padräo }}}\right) * 1000$ 
onde $\mathrm{R}_{\text {amostra }}$ é a razão isotópica ${ }^{13} \mathrm{C} /{ }^{12} \mathrm{C}$ da amostra e $\mathrm{R}_{\text {padrão }}$ é a razão isotópica ${ }^{13} \mathrm{C} /{ }^{12} \mathrm{C}$ do padrão. O padrão utilizado internacionalmente é a rocha calcárea (PDB) da formação Peedee do Grand Canyon nos Estados Unidos.

A base de variação isotópica em plantas resulta do fracionamento isotópico durante a fotossintese. As plantas terrestres podem ser divididas em três grupos fotossintéticos principais, cada um com seu padrão de fracionamento isotópico específico: C3, C4 e MAC (metabolismo ácido das crassuláceas). A composição isotópica destes diferentes tipos fotossintéticos são em geral resultado de: (1) propriedades bioquímicas da fixação primária de $\mathrm{CO}_{2}$ por ação enzimática; (2) limitações da difusão do $\mathrm{CO}_{2}$ dentro das folhas [2] e (3) relação entre a pressão interna de $\mathrm{CO}_{2}$ na câmara estomatal e a pressão externa da atmosfera [7]. As plantas com via fotossintética C3 (ex.: uva, arroz, maçã, cevada, etc.), reduzem $\mathrm{o} \mathrm{CO}_{2}$ para fosfoglicerato (um composto de três carbonos) através da enzima RuBP-carboxilase. Esta enzima discrimina $\mathrm{O}^{13} \mathrm{CO}_{2}$, resultando em valores de $\delta^{13} \mathrm{C}$ relativamente baixos, entre -32 e -23\%o, com média de $-27 \%$ o [2]. Por outro lado, plantas C4 (ex.: canade-açúcar, milho, sorgo, etc.) reduzem o $\mathrm{CO}_{2}$ a ácido aspártico ou ácido málico, ambos compostos com quatro carbonos, através da enzima PEP-carboxilase. Esta enzima não discrimina $0{ }^{13} \mathrm{C}$ como a RuBP-carboxilase, desta forma plantas $\mathrm{C} 4$ têm valores de $\delta^{13} \mathrm{C}$ relativamente mais altos. Tais valores variam entre -15 e $-9 \%$, com média de -13\%o [2]. Assim, as espécies C3 e C4 têm valores distintos de $\delta^{13} \mathrm{C}$, que não se sobrepõem, sendo possivel, portanto, a utilização desses valores na determinação da fonte de carbono.

A determinação da razão isotópica permite uma comparação dos valores de $\delta^{13} \mathrm{C}$ das fontes com o valor de $\delta^{13} \mathrm{C}$ do produto. Assim, quando existem somente duas fontes isotopicamente distintas, o valor de $\delta^{13} \mathrm{C}$ do produto refletirá a quantidade das duas fontes presentes no produto, que pode ser quantificado pela equação de mistura ou diluição isotópica:

$\% C_{3}=\frac{\delta^{13} C_{\text {amostra }}-\delta^{13} C_{4}}{\delta^{13} C_{3}-\delta^{13} C_{4}}$

onde, $\delta^{13} \mathrm{C}_{3}$ e $\delta^{13} \mathrm{C}_{4}$ são a composição isotópica de plantas $\mathrm{C} 3$ e $\mathrm{C} 4$, respectivamente.

No Laboratório de Ecologia Isotópica do Centro de Energia Nuclear na Agricultura (USP) foram analisadas 22 amostras de cerveja, 14 de vinho, 7 de vodka e 20 de café, que foram adquiridas em estabelecimentos comerciais entre agosto e setembro de 2000.

A composição isotópica destas amostras foi determinada em um espectrômetro de massa de razão isotópica (IRMS) Finnigan MAT Delta Plus. No momento da análise, os frascos de cervejas, vinhos e vodkas foram abertos e uma alíquota coletada diretamente, por meio de um capilar de vidro. A seguir, metade do volume do capilar foi recebido em uma cápsula de estanho contendo o fundo coberto por uma pequena quantidade de fase sólida CHROMOSORB W (30 - 60mesh). Logo a seguir, para evitar evaporação, foi realizado o empacotamento apropriado da cápsula e sua colocação imediata no amostrador do analisador elementar (Carlo Erba EA 1110 CHNS). As amostras de café em pó foram pesadas e colocadas também em cápsulas de estanho. Após a oxidação da matéria orgânica presente nas amostras e separação cromatográfica do gás $\mathrm{CO}_{2}$, este foi carreado para o espectrômetro e as concentrações isotópicas do C medidas.

\section{3 - RESULTADOS E DISCUSSÃO}

O café é uma planta do tipo fotossintético C3, portanto, seus valores isotópicos, caso não haja nem um tipo de mistura, devem seguir os valores normalmente encontrados em plantas C3. A maioria dos valores de $\delta^{13} \mathrm{C}$ de amostras de café variaram de $-28,2$ a $-26,1 \%$ (Tabela 1), comparando-se estes valores com o valor de referência encontrado na Tabela 2 , conclui-se que nenhum material de origem $\mathrm{C} 4$ foi misturado ao café. O fato de se ter valores isotópicos em amostras de café que foram cerca de $2 \%$ mais negativos que a referência, pode ser atribuído a diferentes graus de fracionamento isotópico entre $\mathrm{O} \mathrm{CO}_{2}$ atmosférico e as plantas. Este fracionamento distinto entre variedades de plantas de café pode ser atribuído as condições de cultivo, como estresse hídrico, ou diferenças climáticas entre regiões de cultivo. Por exemplo, variedades de café que foram irrigadas tiveram valores de $\delta^{13} \mathrm{C}$ entre $-28,3$ e -26,5\%o, enquanto aquelas submetidas a estresse hídrico tiveram valores de $\delta^{13} \mathrm{C}$ variando entre -26,0 e -24,1\%o [12]. A exceção a este padrão foi a amostra número 20 , que teve um valor de $\delta^{13} \mathrm{C}$ igual a $-16,2 \%$, indicando claramente a presença de plantas de origem $\mathrm{C} 4$ misturadas ao café (Figura 1).

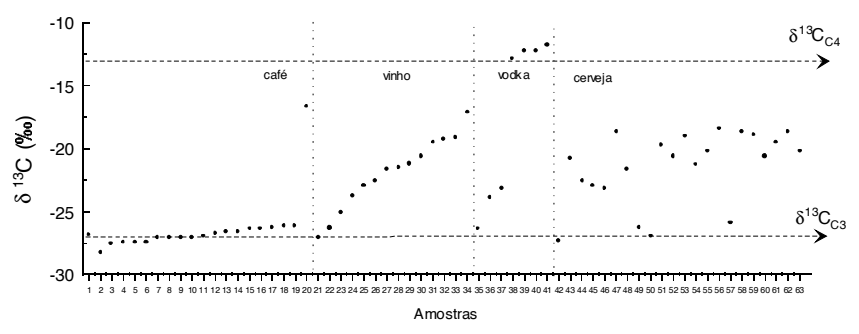

FIGURA 1. Valores isotópicos $\left(\delta^{13} \mathrm{C} \%\right)$ das amostras do presente trabalho. Ver Tabela 1 para detalhes sobre cada amostra. As duas linhas pontilhadas horizontais mostram os valores padrões de $\delta^{13} \mathrm{C}$ para plantas C3 e C4.

De acordo com a Associação Brasileira da Indústria de Café (ABIC), a legislação brasileira não permite nenhum tipo de mistura no pó-de-café comercializado, e aceita apenas $1 \%$ de impureza dos grãos, que consiste na mistura da casca do próprio fruto no processo de moagem. Apesar disto, os resultados de testes de qualidade realizados por esta associação detectaram adulteração pela adição de até $70 \%$ de milho em algumas marcas de café [1]. Baseando-se nos valores isotópicos en- 
contrados, e utilizando-se a equação (2), estimamos que a amostra 20 teve aproximadamente $70 \%$ de plantas $\mathrm{C} 4$ em sua composição. Apesar de discriminar na embalagem a adição de amido, a empresa não discriminou o percentual de amido presente na mistura.

Nas amostras de vinho, dois grupos foram distinguidos: as amostras de vinho produzidas em outros países (amostras 21 e 22) e as produzidas no país. As amostras importadas tiveram valores de $\delta^{13} \mathrm{C}$ mais negativos $(-27,1$ e $-26,3 \%$ ) enquanto as nacionais variaram entre $-25,1$ e -17,1\%o (Tabela 1). Esta distinção provavelmente é devida ao fato de que no processo de fabricação do vinho, segundo a legislação brasileira, pode ser adicionado até $30 \%$ de açúcar de cana ao mosto antes da fermentação, processo conhecido como chaptalização. Por exemplo, baseando-se nesse percentual e utilizando-se a equação (2) e os valores de referência da Tabela 2 , estima-se que um vinho contendo $30 \%$ de açúcar em seu mosto, deveria ter uma composição isotópica de -22,5\%o. Valores isotópicos muito acima deste indicariam uma adição maior de açúcar no mosto. É interessante notar que foi observada uma relação inversa $\left(p>0,01 ; R^{2}=0,52\right)$ entre o preço das amostras de vinho e valores correspondentes de $\delta^{13} \mathrm{C}$, indicando que quanto maior o teor de açúcar adicionado, menor o preço do vinho (Figura 2). O mesmo tipo de relação foi encontrado em amostras de brandies brasileiros [13].

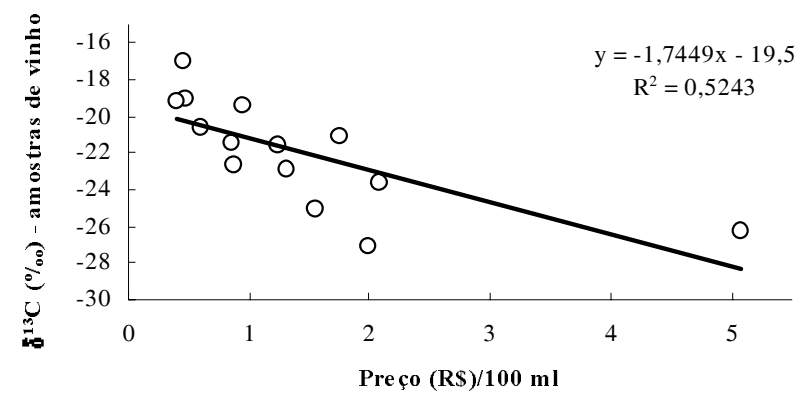

FIGURA 2. Relação entre valores de $\delta^{13} \mathrm{C}$ e preço de aquisição dos vinhos.

A vodka é uma bebida alcoólica destilada produzida a partir do amido de fonte variável. Embora o centeio seja originalmente utilizado na fabricação da vodka, o processo também emprega outros cereais, como mi1ho, arroz e trigo. A batata também é utilizada, assim como em pequenas proporções são utilizados açúcares fermentáveis, tais como melaço de cana ou beterraba [17]. Os valores isotópicos obtidos para as diferentes marcas de vodka variaram entre -26,4 e -11,8\%o (Figura 1). As marcas de vodka importadas apresentaram valores isotópicos característicos de plantas C3 ou com pequena participação de C4 (Tabela 1). Por outro lado, entre as marcas brasileiras, a maioria delas apresentaram valores isotópicos estritamente de plantas $\mathrm{C} 4$, variando seus valores isotópicos entre -12,8 e -11,8\%o (Tabela 1). Apenas a amostra 37 teve um valor isotópico intermediário entre plantas $\mathrm{C} 3$ e $\mathrm{C} 4\left(\delta^{13} \mathrm{C}=-23,1 \%\right.$ ), indicando uma mistura desses dois tipos de plantas na sua composição.

TABELA 1. Valores de $\delta^{13} \mathrm{C}(\%)$, procedência e preço em amostras de bebidas, onde: o preço do café por $100 \mathrm{~g}$ e o preço das demais bebidas por $100 \mathrm{~mL}$.

\begin{tabular}{|c|c|c|c|c|}
\hline & Descricão & Origem & Preco (RS) & $\delta^{13} \mathrm{C}$ \\
\hline \multicolumn{5}{|c|}{$\mathrm{N}^{0} \quad$ Amostras de Café } \\
\hline 1 & Canecão & Campinas (SP) & 1.00 & -26.8 \\
\hline 2 & Do Norte & Manaus (AM) & 0,58 & $-28,2$ \\
\hline 3 & Carrefour & Franca (SP) & 0,62 & $-27,5$ \\
\hline 4 & Manaus & Manaus (AM) & 0,70 & $-27,4$ \\
\hline 5 & Tropical & Barueri (SP) & 0,55 & $-27,4$ \\
\hline 6 & Londrina & Londrin a (PR) & 0.58 & $-27,4$ \\
\hline 7 & Maratá & Lagarto (SE) & 0,55 & $-27,1$ \\
\hline 8 & Do Ponto & Barueri (SP) & 0,65 & $-27,1$ \\
\hline 9 & Minas Rio & B elo Horizonte (MG) & 0,57 & $-27,1$ \\
\hline 10 & Garçonete & Manaus (AM) & 0,60 & $-27,1$ \\
\hline 11 & Morro Grande & Piracicaba (SP) & 0,63 & $\begin{array}{l}-26,9 \\
-26,1\end{array}$ \\
\hline 12 & Sabor Minas & Contagem (MG) & 0,49 & $-26,7$ \\
\hline 13 & 3 Corações & Santa Luzia (MG) & 0,58 & $-26,6$ \\
\hline 14 & Petinho & Recife (PE) & 0,65 & $-26,6$ \\
\hline 15 & Lido & Prudentópolis (PR) & 0,64 & $-26,4$ \\
\hline 16 & Odebrecht & Londrina (PR) & 0,63 & $-26,4$ \\
\hline 17 & Minhouro & Manaus (AM) & 0,65 & $-26,2$ \\
\hline 18 & União & São Paulo (SP) & 0,71 & $-26,1$ \\
\hline 19 & São Paulo & Limoeiro (PE) & 0,39 & $-26,1$ \\
\hline \multirow[t]{2}{*}{20} & Opção & Chorrochó (BA) & 0,34 & $-16,6$ \\
\hline & Amostras de Vinho & & & \\
\hline 21 & Concha Y Toro & Chile & 1,86 & $-27,1$ \\
\hline 22 & Chianti Rufino & Itália & 4,73 & $-26,3$ \\
\hline 23 & Marcus James & B rasil & 1,44 & $-25,1$ \\
\hline 24 & B aron de Lantier Dourado & B rasil & 1,94 & $-23,7$ \\
\hline 25 & Almaden & Brasil & 1,23 & $-22,9$ \\
\hline 26 & San Germain & Brasil & 0,83 & $-22,6$ \\
\hline 27 & B aron de Lantier Branco & B rasil & 1,16 & $-21,6$ \\
\hline 28 & Bella Aurora - São Roque & Brasil & 0,80 & $-21,5$ \\
\hline 29 & $\begin{array}{l}\text { Miolo Seleção } \\
\text { a }\end{array}$ & B rasil & 1,64 & $-21,2$ \\
\hline 30 & Piagentini & Brasil & 0,57 & $-20,6$ \\
\hline 31 & Chateau Duvalier & $\begin{array}{l}\text { Brasil } \\
\text { B ras }\end{array}$ & 0,88 & $-19,5$ \\
\hline 32 & $\begin{array}{l}\text { Chapinha } \\
\text { Salle }\end{array}$ & B rasil & 0,37 & $-19,2$ \\
\hline 33 & Sangue de Boi & $\begin{array}{l}\text { B rasil } \\
\text { B }\end{array}$ & 0,44 & $-19,1$ \\
\hline \multirow[t]{2}{*}{$\begin{array}{l}53 \\
34\end{array}$} & Dom Bosco & Brasil & 0,42 & $-17,1$ \\
\hline & Amo stra s de Vodka & & & \\
\hline 35 & Wiborowa & Polônia & 4.34 & $-26,4$ \\
\hline 36 & Golernatokskaba & Rússia & 3.67 & $\begin{array}{l}-23,4 \\
-23,9\end{array}$ \\
\hline 37 & Polanski & Brasil & 0.37 & $-23,1$ \\
\hline 38 & Sputinik & Brasil & 0.58 & $-12,8$ \\
\hline 39 & Orloff & B rasil & $\begin{array}{l}1.08 \\
1.08 \\
\end{array}$ & $-12,3$ \\
\hline 40 & Baikal & Brasil & 0.55 & $-12,3$ \\
\hline \multirow[t]{2}{*}{41} & Smirnoff & Brasil & 1.16 & $-11,8$ \\
\hline & Marcas de Cervẹj a & & & \\
\hline 42 & $\begin{array}{l}\text { Guiness } \\
\text { G }\end{array}$ & Irlanda & 1.07 & $-27,3$ \\
\hline 43 & Sapporo & Japão & 1.21 & $-20,8$ \\
\hline 44 & Royal Dutch & Holanda & 1.03 & $-22,6$ \\
\hline 45 & Dutch Gold & Holanda & 0.97 & $-22,9$ \\
\hline 46 & Phoenix & Holanda & 0.97 & $-23,1$ \\
\hline 47 & Sol & México & 0.62 & $\begin{array}{l}-18,7 \\
\end{array}$ \\
\hline 48 & Corona & México & 0.57 & $-21,6$ \\
\hline 49 & Urquell & Rep.Tcheca & 0.72 & $-26,2$ \\
\hline 50 & Dab & Alemanha & 0.29 & $-27,0$ \\
\hline 51 & Caracu & Brasil & 0.26 & $-19,7$ \\
\hline 52 & Krill & B rasil & 0.16 & $-20,6$ \\
\hline 53 & Lecker & B rasil & 0.16 & $-19,0$ \\
\hline 54 & Cintra & $\begin{array}{l}\text { B rasil } \\
\text { B rasin }\end{array}$ & 0.16 & $\begin{array}{l}-1,0 \\
-21,3\end{array}$ \\
\hline 55 & Kronenbier & B rasil & 0.26 & $-20,2$ \\
\hline 56 & Bohemia & Brasil & 0.25 & $-18,4$ \\
\hline 57 & Heineken & B rasil & 0.26 & $\begin{array}{l}-10,4 \\
-25,9\end{array}$ \\
\hline 58 & Schincariol & B rasil & 0.17 & $-18,7$ \\
\hline 59 & Antartica & B rasil & 0.19 & $-18,9$ \\
\hline 60 & Skol & B rasil & 0.19 & $\begin{array}{l}-1,20,6 \\
-20,6\end{array}$ \\
\hline 61 & B rahma & B rasil & 0.17 & $-19,5$ \\
\hline 62 & Bavaria & B rasil & 0.17 & $-18,6$ \\
\hline 63 & Kaiser & B rasil & 0.15 & $-20,2$ \\
\hline
\end{tabular}

Por sua vez, a cerveja, segundo as Normas Técnicas Relativas a Alimentos e Bebidas [3], é um produto obtido através da fermentação alcoólica produzida pela levedura Saccharomyces cerevisiae, de um mosto preparado com cevada (planta C3) maltada, lúpulo, água, adicionado ou não de açúcares ou xaropes de cana, beterraba, arroz e milho. As amostras de cervejas brasileiras apresentaram $\delta^{13} \mathrm{C}$ entre $-25,9$ e $-18,4 \%$ (Tabela 1$)$, denotando, portanto, uma contribuição variável de plantas do tipo C4. A presença de plantas $\mathrm{C} 4$ em cervejas parece ser uma constante mesmo em outros países. Das nove cervejas importadas, somente a amostra 42 apresentou 
sinal isotópico típico de plantas C3 (Tabela 1). Os valores isotópicos das demais amostras indicaram que estas, como a maioria das cervejas nacionais, são compostas por uma mistura de fontes $\mathrm{C} 3$ e $\mathrm{C} 4$, com a contribuição de $\mathrm{C} 4$ podendo chegar até $60 \%$ em alguns casos. É interessante notar que, somente quatro amostras de cerveja $(44,45,46$ e 47) declaram a presença de milho (C4) em sua composição.

TABELA 2. Valores de $\delta^{13} \mathrm{C}$ das referências utilizadas para cálculo de diluição isotópica.

\begin{tabular}{|c|c|c|c|}
\hline Produto & $\delta^{13} \mathrm{C} 3$ & $\delta^{13} \mathrm{C} 4$ & Referência \\
\hline Café & $-26,8$ & $-13,0$ & C3: Amostra certificada em grãos \\
\hline Vinho & $-26,3$ & $-11,8$ & $\begin{array}{l}\text { C3: Vinho de origem italiana, onde a adição de açúcar é proibida por } \\
\text { lei. } \\
\text { C4: o valor de melaço da cana-de-açúcar determinado por ROSSI } e t a l \text {. } \\
\text { [14]. }\end{array}$ \\
\hline Vodka & $-26,4$ & $-11,8$ & $\begin{array}{l}\text { C3: vodka polonesa } \\
\text { C4: o valor de melaço da cana-de-açúcar determinado por ROSSI et al. } \\
\text { [14]. }\end{array}$ \\
\hline Cerveja & $-27,3$ & $-11,8$ & $\begin{array}{l}\text { C3: cerveja irlandesa } \\
\text { C4: o valor de melaço da cana-de-açúcar determinado por ROSSI et al. } \\
\text { [14]. }\end{array}$ \\
\hline
\end{tabular}

\section{4 - CONCLUSÕES}

A metodologia de isótopos estáveis mostrou ser uma ferramenta útil para a identificação da origem do material e porcentagem de plantas C3 e C4 na composição de tipos de café e várias bebidas. A Figura 1 demonstra claramente que amostras de várias bebidas contém uma quantidade apreciável de material oriundo de plantas C4. Em alguns casos, como o do café, a presença de material C4 definitivamente altera o gosto da bebida e não é regularmente permitido. Por outro lado, para as bebidas em que a legislação permite uma composição variada, como cerveja e vodka por exemplo, a metodologia isotópica pode ser útil para detectar a presença de ingredientes não especificados no rótulo, mas que seria conveniente ou justo que o consumidor estivesse a par de sua presença.

\section{REFERÊNCIAS}

[1] ABIC. 2000. http://www.abic.com.br/selodepureza/ index.html, Set/15/2000.

[2] BOUTTON, T.W. Stable carbon isotope ratios of natural materials: II. Atmospheric, terrestrial, marine, and freshwater environments. In: COLEMAN, D.C.; FRY, B. (Ed.) Carbon isotope techniques. New York: Academic Press, 1991. cap.11, p. 173-185.

[3] BRASIL. Leis, Decretos, etc. Decreto $n^{\circ} 55871$, de 5 de março de 1965. Diário Oficial da União, Brasília, p. 231232. 1965

[4] BROOKES, S.T.; BARRIE, A.; DAVIES, J.E. A rapid ${ }^{13} \mathrm{C} /{ }^{12} \mathrm{C}$ test for determination of corn syrups in honey. Journal Association Analytical Chemistry, v. 74, n. 4, p. 627629, 1991.

[5] DURST, R.W; WROLSTAD, R.E.; KRUEGER, D.A. Sugar, nonvolatile acid, ${ }^{13} \mathrm{C} /{ }^{12} \mathrm{C}$ ratio, and mineral analysis for determination of the authenticity and quality of red raspberry juice composition. Journal of AOAC international, v. 78, n. 5, p. 1195-1204. 1995.

[6] EUROPEAN COMMUNITY. Directive 2676/90, September 17, 1990.

[7] FARQUHAR, G.D.; EHLERINGER, J.R.; HUBICK, K.T. Carbon isotope discrimination and photosynthesis. Annual Review of Plant Physiology and Plant Molecular Biology. v. 40, p. 503-537, 1989.

[8] GIMÉNEZ-MIRALLES, J.E.; SALAZAR, D.M.; SOLANA, I. Regional origin assignment of red wines from Valencia (Spain) by ${ }^{2} \mathrm{H}$ NMR and ${ }^{13} \mathrm{C}$ stable isotope analysis of fermentative ethanol. Journal Agricultural Food Chemistry, v. 47, p. 2645-2652, 1999.

[9] GUILlou, C.; KOZIET, J.; ROSSMANN, A.; MARTIN, G.J. Determination of the $\mathrm{d}^{13} \mathrm{C}$ content of organic acids and sugars in fruit juices: an inter-comparation study. Analytica Chimica Acta, v. 388, p. 137-143, 1999.

[10] MARTIN, G.J.; GUILLOU, C.; MARTIN, M.L.; CABANIS, M.T.; TEP, Y.; AERNY, J. Natural factors of isotope fractionation and the characterization of wines. Journal Agricultural Food Chemistry, v. 36, p. 316-322, 1988.

[11] MARTIN, G.G; HANOTE, V.; LEES, M.; MARTIN, Y-LOIC. Interpretation of combines ${ }^{2} \mathrm{H}$ SNIF/MNR and ${ }^{13} \mathrm{C}$ SIRA/ MS analyses of fruit juices to detect added sugar. Journal of AOAC international, v. 79, n. 1, p. 62-72, 1996.

[12] MEINZER, F.C.; GOLDSTEIN, G.; GRANTZ, D.A. Carbon isotope discrimination in coffee genotypes grown under limited water supply. Plant Physiology, v. 92, p. 130135, 1990.

[13] PISSINATTO, L.; MARTINELLI, L.A.; VICTÓRIA, R.L.; CAMARGO, P.B.; CHEDID, J.J. Using stable carbon isotopic analyses to access the botanical origin of ethanol in Brazilian brandies. Food Research International, v. 32, p. 665-668, 1999.

[14] ROSSI, N.F.; MARTINELLI L.A.; LACERDA, T.H.M.; CAMARGO, P.B.de; VICTORIA, R.L. Análise da adulteração de méis por açúcares comerciais utilizando-se a composição isotópica do carbono. Ciênc. Tecnol. Aliment., v. 19, n. 2, p. 199-204, 1999.

[15] ROBMANN, A.; SCHIMDT, H.L.; RENIERO, F.; GIUSEPPE, V.; MOUSSA, I.; MERLE, M.H. Stable carbon isotope content in ethanol of EC data bank wines from Italy, France and Germany. Z Lebensm Unters Forsch, v. 203, p. 293-301, 1996.

[16] SIMPKINS, W.A.; PATEL, G.; HARRISON, M.; GOLDBERG, D. Stable carbon isotope ratio analysis of Australian orange juices. Food Chemistry, v. 70, p. 385-390, 2000.

[17] VARNAM, A.H; SUTHERLAND, J.P. Beverages Technology, chemistry and microbiology. London: Chapman and Hall, 1994. 464p.

[18] WEBER, D.; ROBMANN, A.; SCHWARZ, S.; SCHIMDT, H.L. Correlations of carbon isotope ratios of wine ingredients for the improved detection of adulterations: I. Organic acids and ethanol. Z Lebensm Unters Forsch - A, v. 205, p. 158-164, 1997.

[19] WHITE JR, J.W. ; DONER, L. The ${ }^{13} \mathrm{C} /{ }^{12} \mathrm{C}$ ratio in honey. Journal of Apicultural Research, v. 17, n. 2, p. 94-99, 1978.

[20] WHITE JR, J.W. ; ROBINSON, F.A. ${ }^{13} \mathrm{C} /{ }^{12} \mathrm{C}$ ratios of citrus honeys and nectars and their regulatory implications. Journal Association Analytical Chemistry, v. 66, n. 1, p. 1-3, 1982. 